\title{
Hemangioendotelioma epitelioide hepático de presentación como lesión focal incidental única: reporte de caso
}

\section{Hepatic epithelioid hemangioendothelioma of presentation as single focal incidental lesion: case report}

\author{
Juan Roberto Padilla Flores ${ }^{1}$, Jhonathan Percy Quispe Huachaca ${ }^{2}$, Herbert Alejandro Yabar \\ Berrocal $^{3}$, Nadia Aracelli Malpartida Beraún ${ }^{4}$ \\ ${ }^{1}$ Médico Asistente, Servicio de Radiodiagnóstico, Hospital Nacional Edgardo Rebagliati Martins, EsSalud, Lima, Perú. \\ ${ }^{2}$ Médico Residente, Servicio de Radiodiagnóstico, Hospital Nacional Edgardo Rebagliati Martins, EsSalud, Lima, Perú \\ ${ }^{3}$ Médico Asistente, Servicio de Anatomía Patológica, Hospital Nacional Edgardo Rebagliati Martins, EsSalud, Lima, Perú. \\ ${ }^{4}$ Médico Residente Servicio de Gastroenterología Clínica, Hospital Nacional Edgardo Rebagliati Martins, EsSalud, Lima, Perú.
}

Correspondencia

Juan Roberto Padilla Flores

jjuanpadilla01@hotmail.com

Recibido: $1^{\circ}$ de junio 2017

Aceptado: 26 de junio 2017

Conflictos de interés: Los autores declaran no tener conflictos de interés

Financiamiento: Autofinanciado

Citar como: Padilla Flores JR, Quispe Huachaca JP, Yabar Berrocal HA, Malpartida Beraún NA. Hemangioendotelioma epitelioide hepático de presentación como lesión focal incidental única: reporte de caso. An Fac med. 2017;78(4):424-9

DOI: http://dx.doi.org/10.15381/

anales.v78i4.14265
An Fac med. 2017;78(4):424-9/ http://dx.doi.org/10.15381/anales.v78i4.14265

\section{Resumen}

El hemangioendotelioma epitelioide hepático (HEH) es un tumor vascular maligno poco frecuente, de origen endotelial, de lenta progresión y de bajo grado de malignidad. Suele presentarse con mayor compromiso en mujeres, sin una etiología conocida. La clínica es variable, desde casos asintomáticos hasta dolor abdominal. Las características imagenológicas más frecuentes son lesiones nodulares hepáticas coalescentes, de tamaño variable, que muestran apariencia de "lesión en diana" en tomografía computarizada (TC) y resonancia magnética (RM), con retracción capsular. El principal diagnóstico diferencial son las metástasis hepáticas. El curso evolutivo es variable e imprevisible, con una mortalidad posterior al diagnóstico de hasta el $50 \%$ de los pacientes. Las modalidades terapeúticas incluyen la extirpación quirúrgica de la lesión y el trasplante hepático como opciones más útiles, además de la quimioterapia regional/sistémica y tratamiento inmunológico.

El presente caso describe las principales características clínico-patológicas de este raro tumor, con la particularidad de su forma de presentación como lesión focal incidental única, además de la modalidad terapéutica utilizada (extirpación quirúrgica) en este tipo de lesión.

Palabras clave. Hemangioendotelioma epitelioide hepático; Tumores hepáticos; Lesión focal hepática

\section{Abstract}

Hepatic epithelioid hemangioendothelioma $(\mathrm{HEH})$ is a very rare malignant vascular tumor of endothelial origin, of slow progression and low malignity degree. It is more common in women, of unknown etiology. Clinical manifestations of $\mathrm{HEH}$ are non-specific, from asymptomatic to abdominal pain.

The more frequent radiological features are nodular hepatic lesions, of variable size, which show appearance of "target sign" in computed tomography (CT) and magnetic resonance imaging (MRI), with capsular retraction. Hepatic metastases are the principal differential diagnosis. The clinical course is variable, with a mortality rate to the diagnosis of up to $50 \%$ of the patients.

The therapeutical options include the surgical extirpation (removal) of the tumor and hepatic transplant as the more useful, besides the regional /systemic chemotherapy and immunological treatment. The present case describes the principal clinical - pathological characteristics of this rare tumor, with the particularity of its form of presentation as focal lesion, besides the therapeutical modality used (surgical removal) in this type of illness.

Keywords. Hepatic epithelioid hemangioendothelioma; Liver tumors; Liver focal lesion 


\section{INTRODUCCIÓN}

El hemangioendotelioma epitelioide es un tumor poco frecuente, cuya localización hepática es muy rara en el adulto. Descrita por primera vez como lesión hepática en $1984^{(1)}$, su etiología es desconocida, presentando una sintomatología inespecífica. En la actualidad, los estudios anatomopatológicos muestran que es un tumor vascular maligno de origen endotelial y de bajo grado de malignidad, siendo su pronóstico y curso variable, aunque con una tasa de sobrevida mayor con respecto a otros tumores vasculares malignos hepáticos como el angiosarcoma.

Este tumor representa un reto diagnóstico mayor, debido a que su presentación típica es similar a las metástasis ${ }^{(2)}$, requiriendo la confirmación anatomopatológica, en la mayoría de casos. Los métodos de diagnóstico por imagen ayudan a valorar la estadificación del estadio clínico inicial de presentación y el planeamiento terapéutico ${ }^{(3)}$.

El presente estudio describe las características imagenológicas y clínicopatológicas de un caso de hemangioendotelioma epitelioide hepático primario, confirmado con estudio inmunohistoquímico realizado por biopsia excisional, con características singulares, como que el afectado sea un paciente varón, su presentación asintomática y, sobre todo, el hallazgo radiológico de lesión focal única en el parénquima hepático. Además, se menciona la terapéutica exitosa mediante la extirpación quirúrgica de la lesión solitaria en casos de esta presentación.

\section{CASO CLÍNICO}

Varón de 48 años, natural y procedente de Lima-Perú, con antecedentes médicos de tuberculosis pulmonar ya curada hace 20 años y litiasis renal bilateral asintomática, sin antecedentes de importancia, quirúrgicos o de otra índole. Acude a consulta externa con un tiempo de enfermedad de aproximadamente 1 año, caracterizado por dolor tipo cólico, de leve intensidad, localizado en región lumbar derecha, de presentación intermitente, no relacionado con la ingesta de alimen-

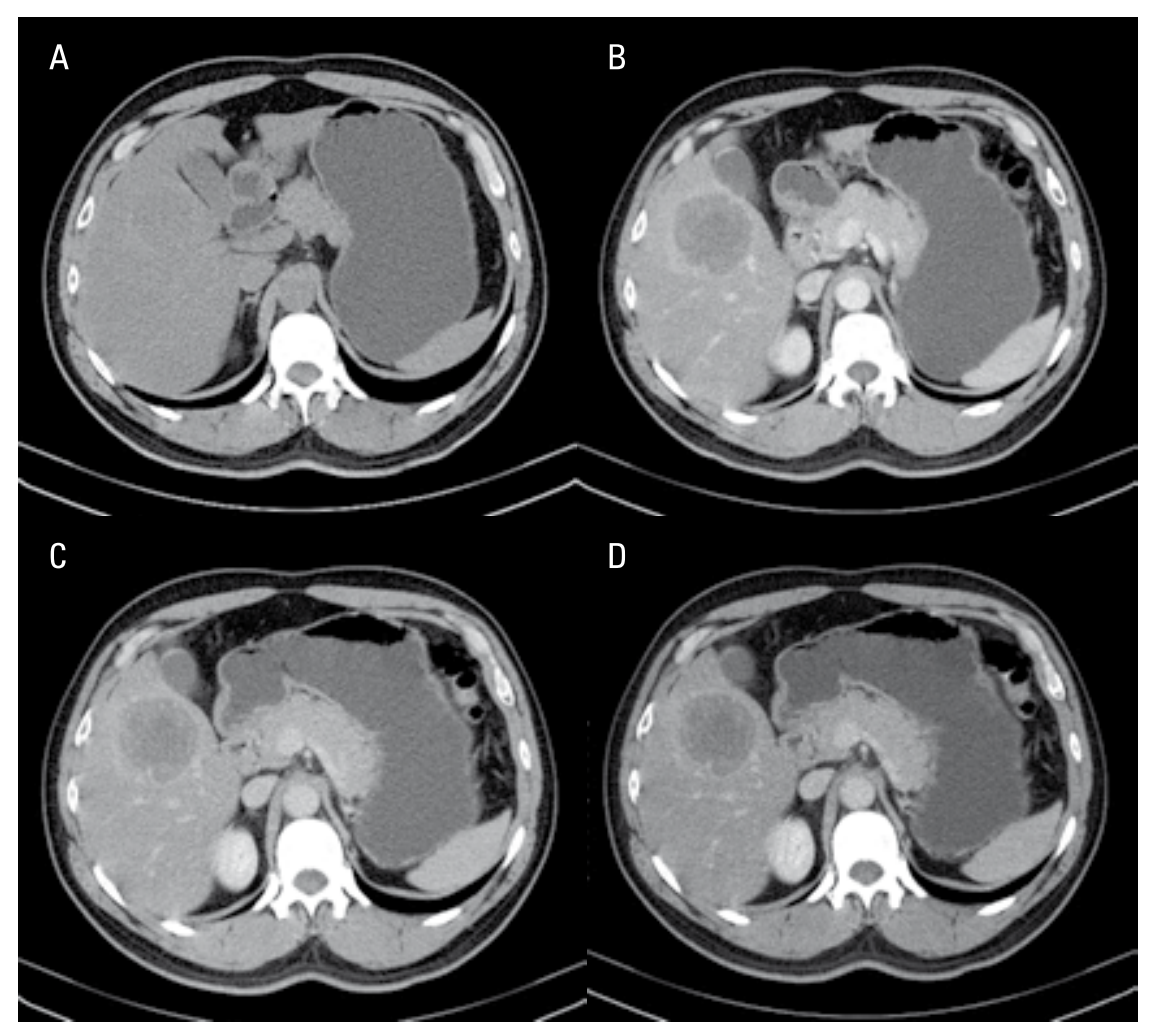

Figura 1. Evaluación por TC abdominal con contraste en la fase no contrastada (A), arterial (B), venosa (C) y tardía (D).

tos. Es evaluado en consultorio externo y se le realizó una ecografía renal y abdominal (por el antecedente de litiasis y la sospecha de un cuadro de cólico renal asociado), se evidenció incidentalmente la presencia de una lesión hepática "quística" de $3 \mathrm{~cm}$ en segmento $V$, por lo que se le indicó evaluaciones seriadas de control. Hace 4 meses, en un nuevo control, se evidencia aumento de las dimensiones de esta lesión (5-6 cm), motivo por el cual es referido al Hospital Nacional Edgardo Rebagliati Martins, EsSalud, Lima, Perú, para ampliación de estudios.

En los análisis bioquímicos no se encontró mayor anormalidad, siendo las enzimas hepáticas (TGO-TGP) en valores normales (28 y $59 \mathrm{U} / \mathrm{L}$, respectivamente). La bilirrubina total fue de $1,49 \mathrm{mg} /$ dL y la albúmina $4,7 \mathrm{~g} / \mathrm{dL}$. La serología vírica y los marcadores tumorales estuvieron dentro de rangos normales. Se le realiza una nueva ecografía abdominal, la cual mostró una masa en el hígado con dimensiones de $60 \times 60 \times 60$ mm (diámetro longitudinal $x$ diámetro posterior $x$ diámetro transverso), localizada a nivel del segmento $V$, hipoecogénica, de bordes regulares, con escaso flujo al estudio Doppler color, sin otra alteración en los órganos abdominales.

La tomografía computarizada (TC) abdominal con y sin contraste (figura 1 ) confirmó los hallazgos obtenidos en la ecografía, evidenciando imagen nodular esferoide, con dimensiones de $72 \times 60$ $\mathrm{mm}$, localizada en el segmento $\mathrm{V}$. En la fase no contrastada es de naturaleza hipodensa. En la fase arterial (60") presenta tenue realce de sus límites y del parénquima hepático adyacente, mientras que en la fase portal (90") se aprecia mayor realce periférico y escaso realce al interior de la lesión, además de un tenue anillo hipodenso mal delimitado en la zona más periférica de la lesión. La fase tardía (120") no mostró aclaramiento significativo del realce al contraste (fenómeno 


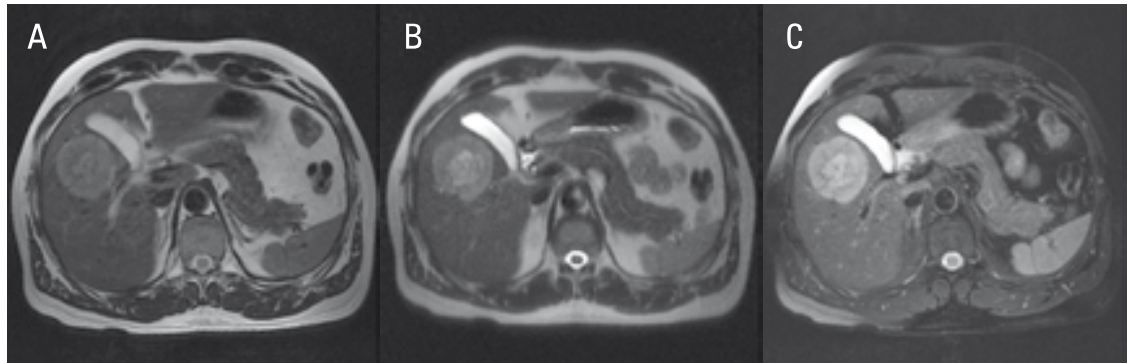

Figura 2. Evaluación por RM abdominal en ponderación T2 (A), T2 HASTE (B) y T2 con saturación grasa (C).

de "wash-out"). Estas características se relacionaron con proceso neoformativo de baja vascularidad. Resto del estudio no mostró otras anormalidades significativas.

Se le realiza, además, una resonancia magnética (RM) con administración de gadolinio, la cual mostró una lesión focal de aspecto nodular, localizada en el segmento $V$, de bordes definidos y regulares. En la ponderación T2, T2 HASTE

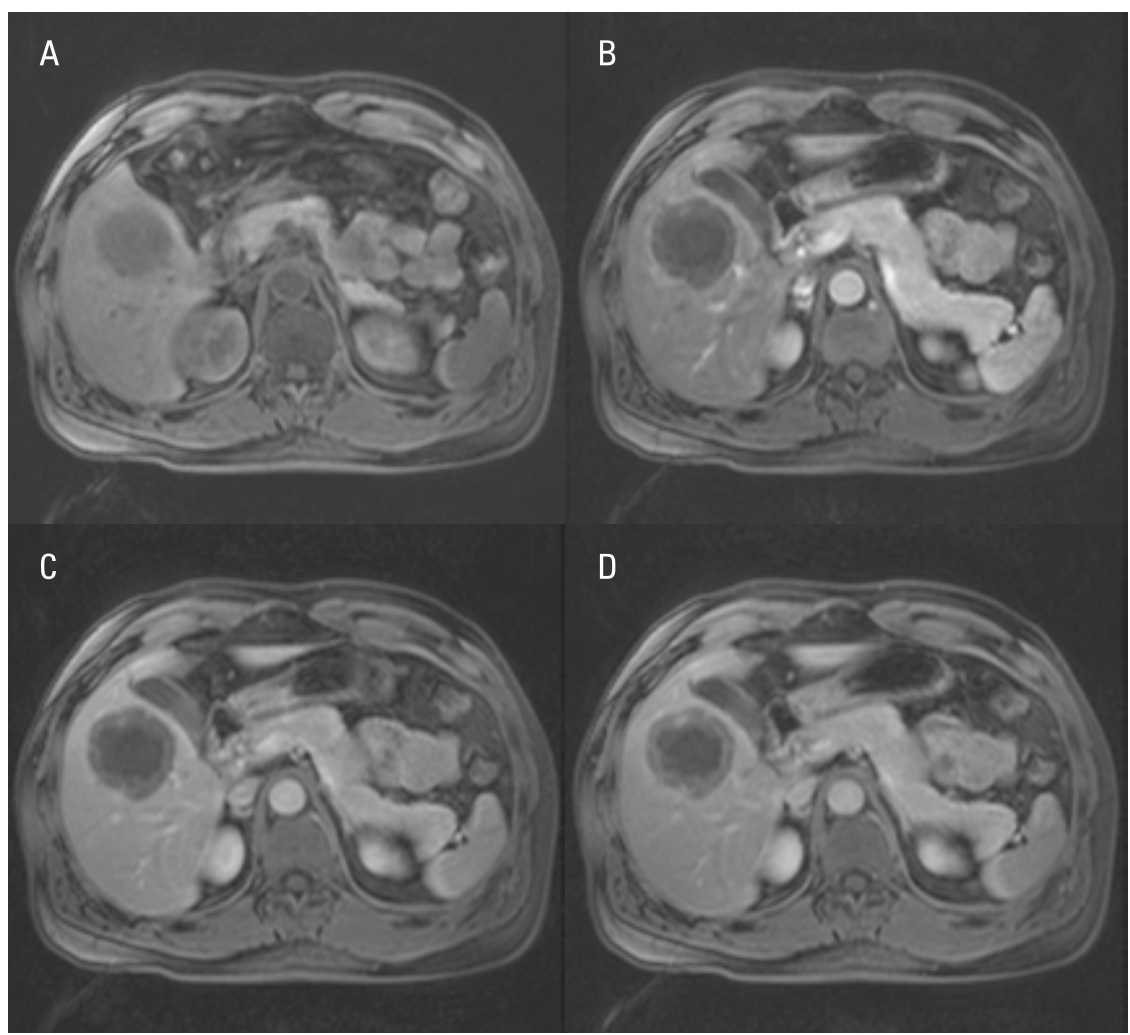

Figura 3. Evaluación por RM abdominal en ponderación T1 con gadolinio y saturación grasa: fase no contrastada (A), fase arterial (B), fase venosa (C) y fase tardia (D).
En la ponderación T1 con gadolinio y saturación grasa (figura 3), secuencias en axial y coronal, mostró realce temprano en la zona periférica, conservando el patrón "en diana", con tres zonas concéntricas: una zona hipointensa central, una zona en anillo grueso con realce al contraste en la periferie cercana y otra zona anular más externa sin significativo realce de poco grosor, observada en la fase más tardía (realce progresivo).

Otros estudios adicionales realizados, incluyeron endoscopía digestiva alta y colonoscopía, cuyos resultados fueron normales.

En cuanto a los estudios para la obtención del diagnóstico específico, se indica la realización de una biopsia hepática percutánea por radiología intervencionista, cuyo resultado no fue concluyente, motivo por el cual es sometido a procedimiento de excisión quirúrgica mediante hepatectomía parcial.

El informe anatomopatológico definitivo de la pieza anatómica (figura 4) describe producto de hepatectomía parcial, especimen que pesa 560 gramos y que mide $19 \times 14 \times 8 \mathrm{~cm}$, con cara cruenta que mide $19 \times 9.5 \mathrm{~cm}$. En los cortes seriados, se evidenció un nódulo que mide $7 x$ $7 \times 7 \mathrm{~cm}$, de bordes definidos, con centro blanquecino, a $1 \mathrm{~cm}$ de la cara cruenta. El estudio histológico de la biopsia excisional (figura 5) mostró células fusiformes epitelioides con pleomorfismo celular y algunas áreas de esclerosis, atrapados en conductillos hepáticos con una matriz central de estroma fibroso y que a los estudios de inmunohistoquímica mostró positividad a CD34, negatividad a CK7 (citokeratina 7) y un ki-67 de 1\%; hallazgos en relación a hemangioendotelioma epitelioide hepático.

Posteriormente, se realizó una TC abdominal de control a los 3 meses de realizada la cirugía, no evidenciando mayor lesión tumoral residual, ni extensión hacia órganos a distancia. Hasta la actualidad, el paciente viene siendo controlado por consultorio externo y con la indicación de TC de seguimientos seriados, sin mayor intercurrencia hasta el momento actual. 


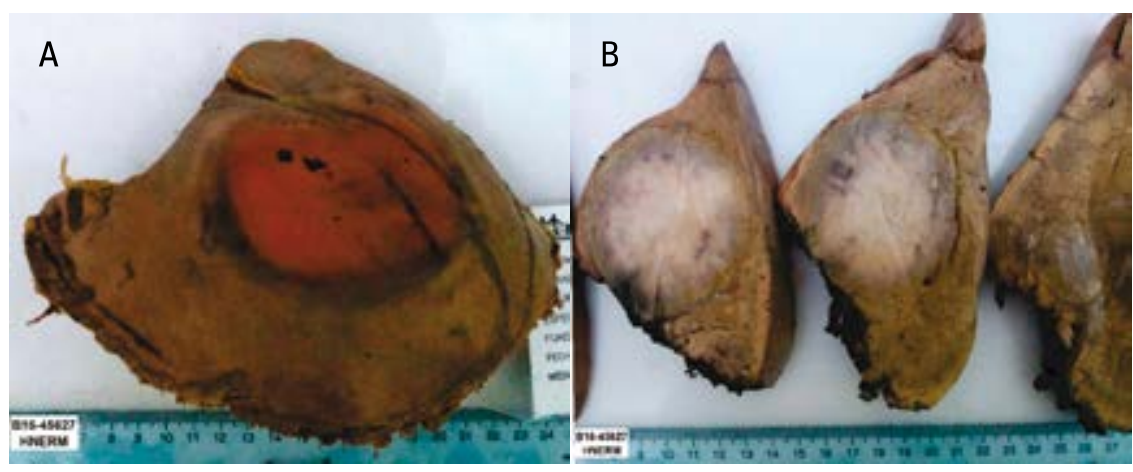

Figura 4. Estudio anatomopatológico. Macroscopia: visión general de la lesión tumoral hepática (A), visión al corte (B).

\section{DISCUSIÓN}

El hemangioendotelioma epitelioide hepático (HEH) es un tumor bastante infrecuente, de una incidencia no conocida con exactitud, aunque algunos reportes estiman un aproximado del 0,1\%, sin exceder el caso por 100,000 habitantes ${ }^{(1)}$. Su origen embrionario deriva de las células endoteliales de la mesénquima tisular; por ello, puede presentar afectación tanto del hígado (órgano más frecuente- mente afectado) como de otros órganos viscerales (cerebro, pulmón, hueso, estómago, bazo, tejidos blandos, etc.)

Este tumor en primera instancia fue reportado por Weiss y Enzinger ${ }^{(2)}$, en 1982, siendo los primeros casos estudiados de localización extrahepática. Ishak ${ }^{(3)}$, en 1984, encontró ciertas características de la enfermedad tumoral hepática, como su presentación más típica en forma de lesiones nodulares múltiples.



Figura 5. Estudio anatomopatológico. Microscopia: tinción con $\mathrm{H}-\mathrm{E}$, a menor (A) y mayor aumento (B). Inmunohistoquímica: tinción con CD 34, a menor (C) y mayor aumento (D).
No hay hasta el momento un factor etiológico seguro conocido, si bien ha sido reportada su asociación con el uso de anticonceptivos orales y la exposición a cloruro de vinilo, además de las hepatitis virales y el consumo excesivo de alcohol (4). Lo anterior se puede asociar a sus aspectos epidemiológicos, dado que afecta más al sexo femenino ${ }^{(5)}$, en una proporción de $2 / 1$ con respecto al sexo masculino, con un rango de edad promedio de 25-58 años ${ }^{(6)}$, y una media de 45 años. El factor genético también tiene cierto papel en su patogenia, ya que se ha descrito una específica translocación cromosómica t (1;3) (p36.3; q25) para el desarrollo de este tumor ${ }^{(7)}$.

El cuadro clínico abarca desde cuadros asintomáticos hasta síntomas y signos poco específicos, siendo los más comunes el dolor abdominal tipo cólico, la ictericia y la hepatoesplenomegalia ${ }^{(8)}$. Asimismo, la analítica sanguínea es inespecífica, siendo lo más llamativo un leve incremento de los niveles séricos de las enzimas hepáticas; los marcadores tumorales hepáticos (alfa fetoproteína y CEA) se encuentran en valores normales ${ }^{(9)}$.

El estudio imagenológico puede ayudar a guiar el diagnostico ${ }^{(10)}$, además de clasificar la enfermedad en dos fenotipos: la forma nodular solitaria y la multinodular, así como establecer tres patrones de crecimiento: nodular, coalescente y mixto ${ }^{(11)}$. El hallazgo más característico es la presencia de múltiples lesiones nodulares o masas hepáticas de localización periférica y con tendencia a la coalescencia, configuración "en diana" (12) y realce al contraste de forma periférica y tardía.

El estudio por ultrasonido (US) muestra nódulos tumorales de ecogenicidad variable, siendo mayormente de tipo hipoecogénico ${ }^{(13)}$. Algunas lesiones isohiperecogénicas pueden mostrar anillos hipoecogénico periféricos, que pueden hacer sospechar el diagnóstico.

Las características más resaltantes a la evaluación por TC no contrastada ${ }^{(14)} \mathrm{com}$ prenden imágenes nodulares hipodensas (focos de atenuación disminuida, por la presencia de estroma mixoide, del parénquima hepático normal) coalescentes de ubicación periférica (en más del 75\% de casos), con retracción de la cápsula (por 
fibrosis circundante e isquemia). El tamaño es variable, desde pequeños nódulos hasta grandes masas confluentes, algunas puedan presentar calcificaciones en su interior. La TC con la administración de contraste ${ }^{(15)}$ evidencia un clásico patrón de realce "en diana", compuesto una parte central que no realza o lo hace tardíamente (estroma mixoide e hialinizado), un anillo periférico interno (vascularidad incrementada) que realza ávidamente, y un halo hipodenso periférico externo (zona avascular).

Las características a la evaluación por RM (12) incluyen: en ponderación T1, las lesiones son hipointensas en su zona central; con un delgado anillo hipointenso periférico. En la ponderación T2, la zona central se observa hiperintensa, conservando el anillo hipointenso periférico. La ponderación T1 con administración de gadolinio muestra también el patrón "en diana" observado en la TC: 3 capas concéntricas con una señal de intensidad alternante, el centro hipointenso, la periferie con una capa interna gruesa, hiperintensa, que realza al contraste y otra capa externa, delgada, sin mayor realce. Los hallazgos radiológicos fueron sugerentes en este caso de un tumor vascular por la forma de captación de contraste, siendo necesaria la confirmación histológica posterior.

Aunque algunos hallazgos radiológicos pueden ser típicos, el diagnóstico solo por los estudios de imagen suele ser difícil debido a que pueden ser similares a los encontrados para las metástasis hepáticas.

Las características anatomopatológicas incluyen al estudio macroscópico nódulos tumorales, con bordes regulares, de color blanquecino o gris pálido, consistencia firme y tamaño variable; la mayoría confluyendo en la periferie ${ }^{(9)}$. Los hallazgos al estudio microscópico comprenden la proliferación sinusoidal de células fusiformes dendríticas o epitelioides, la presencia de una matriz central de estroma mixoide o fibroso, la obliteración de vasos sanguíneos junto a necrosis variable del centro de la lesión y pleomorfismo celular. La inmunohistoquímica es típicamente positiva para marcadores de células endoteliales como el factor VIII (células endoteliales), CD 34 o CD 31 (11); lo que confirma el diagnóstico. En particular, la alta celularidad que se pueda observar es el factor predictivo más significativo para el pronóstico. Con respecto al presente caso, el diagnostico se confirmó con el estudio anatomopatológico consistente con un tumor de origen endotelial e inmunohistoquímica positiva a CD 34.

El diagnóstico diferencial incluye al colangiocarcinoma intrahepático periférico (masa heterogénea con retracción capsular, siendo lo distintivo la presencia de varias lesiones satélites, además de la dilatación biliar intrahepática), el carcinoma hepatocelular o las metástasis (siendo lo distintivo la captación heterogénea de contraste, presencia de modularidad mural o cambios quísticos/necróticos) y el hemangioma (especialmente en asociación con la cirrosis hepática).

Muchas estrategias de tratamiento están disponibles, incluyendo quimioterapia sistémica/regional, radioterapia, hormonoterapia, quimioembolización, termoablación por radiofrecuencia, excisión quirúrgica y trasplante hepático ${ }^{(16)}$. La última opción es la modalidad de tratamiento más utilizada, debido a que la mayoría de pacientes presentan lesiones multifocales al momento del diagnóstico ${ }^{(6)}$, es decir, en casos de HEH irresecable, sin metástasis extrahepáticas, el trasplante hepático es una excelente indicación terapeútica ${ }^{(17)}$, con una sobrevida mucho mejor que en los pacientes trasplantados por hepatocarcinoma avanzado. En nuestro caso, la excisión quirúrgica fue el tratamiento de elección debido a la naturaleza única unilobar de la lesión, sin presentación de metástasis a distancia al momento del diagnóstico y al buen estado funcional del paciente.

El pronóstico depende de la presencia o no del compromiso extrahepático (metástasis al bazo, mesenterio, ganglios peritoneales, pulmón y tejido óseo en el $40 \%$ de casos al momento del diagnóstico), siendo el curso variable e impredecible, con tasas de supervivencia de 1 a 5 años del 80 y $64 \%$, respectivamente ${ }^{(17)}$. Hasta el momento, el paciente viene recibiendo controles periódicos en nuestro hospital con estudios de TC seriados que, realizado el primero a los tres meses después de la cirugía, no muestra mayor recurrencia tumoral ni presencia de lesiones sospechosas de metástasis a distancia, además conservando un buen estado funcional postquirúrgico.

En resumen, el caso que reportamos tiene características interesantes, al considerar un tumor hepático muy raro, no reportado en el medio, además de que su presentación inicial se dio como lesión focal única, por lo que la opción de tratamiento quirúrgica fue la indicada, resultando en una sobrevida exitosa del paciente hasta la fecha.

\section{REFERENCIAS BIBLIOGRÁFICAS}

1. Hertl M, Cosimi AB. Liver transplantation for malignancy. Oncologist. 2005;10:269-281. doi: 10.1634/ theoncologist.10-4-269.

2. Weiss SW, Enzinger FM. Epithelioid hemangioendothelioma: a vascular tumor often mistaken for a carcinoma. Cancer. 1982;50:970-981.

3. Ishak KG, Sesterhenn IA, Goodman ZD, Rabin L, Stromeyer FW. Epithelioid hemangioendothelioma of the liver: a clinicopathologic and follow-up study of 32 cases. Hum Pathol. 1984;15:839-852.

4. Marchiano D, Fisher F, Hofstetter S. Epithelioid hemangioendothelioma of the heart with distant metastases. A case report and literature review. J Cardiovasc Surg. 1993; 34:529-533.

5. Läuffer J, Zimmermann A, Krähenbüh $\mathrm{L}$, Triller J, Baer H. Epithelioid hemangioendothelioma of the liver a rare hepatic tumor. Cancer. 1996;78:2318-2327. doi: 10.1002/ (SICI)1097-0142(19961201)78:11<2318::AIDCNCR8>3.0.CO;2-I.

6. Galletto Pregliasco A, Wendum D, Goumard C Arrivé L. Hepatic epithelioid hemangioendothelioma. Clinics and Research in Hepatology and Gastroenterology. 2016; 40(2):136-8. doi:10.1016/j. clinre.2015.10.002

7. Woelfel C, Liehr T. Molecular cytogenetic characterization of epithelioid hemangioendothelioma. Cancer Genet. 2011;204:671-676. doi: 10.1016/j. cancergen.2011.11.007.

8. Dos Santos G, Collazo C, Maciel G. Hemangioendotelioma epitelioide hepático diseminado: a propósito de un caso. Rev Med Urug. 2011;27:94-97.

9. Kenny AG, Spina JC, Garcia Mónaco RD. Hemangioendotelioma epitelioide hepático: un desafio diagnóstico para el médico radiólogo. Rev Argent Radiol. 2014;78(1):35-41.

10. Azzam RI, Alshak NS, Pham HP. AIRP best cases in radiologic-pathologic correlation: hepatic epithelioid hemangioendothelioma. Radiographics. 2012;32(3):789-94.doi: 10.1148/rg.323115010.

11. Yarze N, Yarze JC. Radiographic and histologic findings of a rare liver tumor-Hepatic Epithelioid Hemangioendothelioma. Dig Dis Sci. 2016;61(6):1178-9. doi: 10.10007/s10620-0164039-z.

12. Hu HJ, Jin $Y W$, Jing QY, Shrestha A, Cheng NS, Li FY. Hepatic epithelioid hemangioendothelioma: 
Dilemma and challenges in the preoperative diagnosis. World J Gastroenterol. 2016 7;22(41): 9247-9250. doi:10.3748/wjg.v22.j41.9247.

13. Lyburn ID, Torreggiani WC, Harris AC, Zwirewich CV, Buckley AR, Davis JE, et al. Hepatic epithelioid hemangioendothelioma: sonographic, CT, and MR imaging appearances. Am J Roentgenol. 2003;180:1359-64. doi: 10.12214/ ajr.180.5.1801359.

14. Soo-Heui Baek, Jung-Hee Yoon. Computed tomography and magnetic resonance imaging findings of a malignant Hepatic Epithelioid Hemangioendothelioma: a rare case of solitary small nodular form. Journal of Investigative Medicine High Impact Case Reports. 2013;1(3):2324709613504549. doi: 10.1177/2324709613504549.

15. Zhou L, Cui M-Y, Xiong J, Dong Z, Luo Y, Xiao H, et al. Spectrum of appearances on CT and MRI of hepatic epithelioid hemangioendothelioma. BMC Gastroenterol. 2015 Jun 19;15(69). doi: 10.1186/ s12876-015-02999-x.

16. Mehrabi A, Kashfi A, Fonouni H, Schemmer P, Sch- mied BM, Hallscheidt P, et al. Primary malignant hepatic epithelioid hemangioendothelioma: a comprehensive review of the literature with emphasis on the surgical therapy. Cancer. 2006;107(9):2108-21. doi: 10.1002/cncr.22225.remisz.

17. Remiszewski P, Szczerba E, Kalinowski P, Gierej B, Dudek K, Grodzicki M, et al. Epithelioid hemangioendothelioma of the liver as a rare indication for liver transplantation. World J Gastroenterol. 2014;20(32):11333-9. doi: 10.3748/wjg.v20. i32.11333 